\section{Bilateral abdominal lipohypertrophy after continuous subcutaneous infusion of insulin}

Continuous subcutaneous infusion of insulin has brought about smooth control of blood glucose concentrations in many patients with brittle diabetes, and few local complications after long-term treatment have been reported. We describe a patient who developed appreciable bilateral abdominal lipohypertrophy at sites of insulin infusion within five months of the start of treatment. To our knowledge this is the first report of this complication.

\section{Case report}

A 58-year-old diabetic woman was referred for stabilisation of diabetes. She had presented initially in 1951 at the age of 27 with ketoacidosis and started on twice-daily injections of insulin. In 1976 diabetic retinopathy was noted, and in 1979 widespread peripheral neuropathy. She developed penetrating ulcers of the left hallux in 1979, and in 1980 required enucleation of the left eye after hypopyon and panophthalmitis. She developed repeated infected ulcers of both feet; the toes were dusky in spite of adequate pedal pulses. A femoral arteriogram in August 1981 confirmed that there was no disease of the large vessels, thereby confirming the clinical impression of the presence of diabetic microvascular disease. One of her toes was amputated. Throughout this period her diabetic control was erratic and characterised by frequent hypoglycaemic episodes, often presenting as coma.

In view of the inadequate diabetic control continuous subcutaneous infusion of insulin was started (monocomponent Actrapid, Novo) via a battery-operated pump (Mill Hill infusor). Insulin was delivered subcutaneously via a butterfly needle (Venisystems Abbott, gauge 25). This resulted in excellent control of her blood glucose concentrations, and her haemoglobin $\mathrm{A}_{1}$ concentration fell from $12 \%$ to $9 \%$.

During the six months after subcutaneous insulin infusion was started she had no further hypoglycaemic episodes. In early May 1982 diabetic control remained good, but she noted soft, painless swellings $10 \times 7 \mathrm{~cm}$ at the sites of the insulin infusion-one on each side of her abdomen (figure). Diabetic control continued to be excellent despite this appreciable lipohypertrophy.

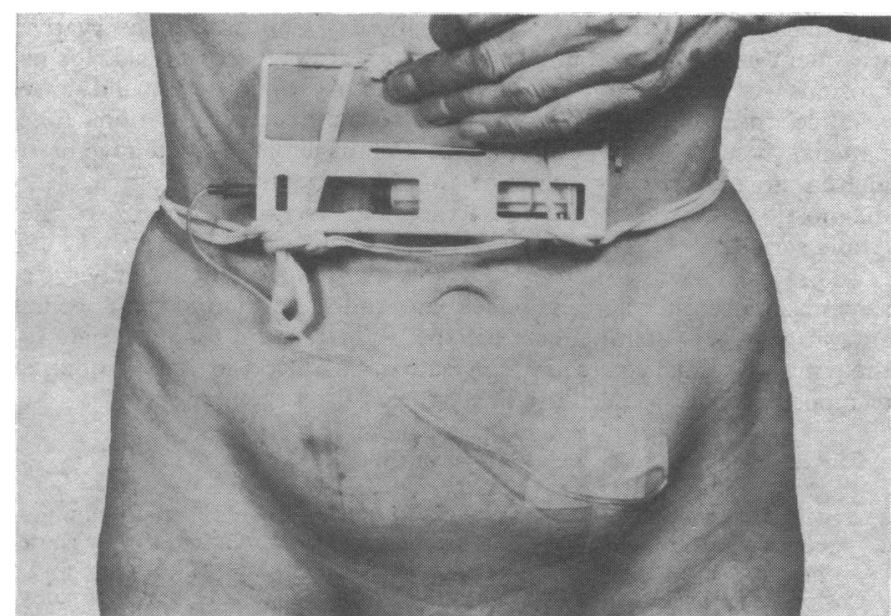

Bilateral abdominal lipohypertrophy due to subcutaneous insulin infusion. The patient was extremely thin.

\section{Comment}

Three important points emerge from this case study. Firstly, subcutaneous insulin infusion is extremely useful and effective in managing insulin-dependent diabetics with recurrent hypoglycaemia. The quality of diabetic control was not altered by the appearance of lipohypertrophy. Secondly, low-dose continuous delivery of insulin does not protect against the development of local lipohypertrophy. In the past it was believed that the injection of boluses of insulin, and the progressive accumulation of an altered or native form of this hormone might play a part in the pathogenesis of lipohypertrophy. ${ }^{1}$ One of the potential advantages of subcutaneous insulin infusion is that clearance of insulin from the site of infusion is closely related to the rate of infusion, thus ensuring smooth control of blood glucose concentrations on the one hand and lack of accumulation of insulin at the infusion site on the other. Thirdly, the rapid development of bilateral lipohyper- trophy within a short time of the start of subcutaneous insulin infusion points to an extraordinary sensitivity of this patient's abdominal subcutaneous fat to insulin. It is noteworthy that this patient had not developed local cutaneous changes during the previous 30 years of her diabetes, when she injected herself in her thigh. Clearly, the sensitivity of subcutaneous fat to insulin in her abdomen and thigh were appreciably different, unless it is argued that it was the combination of insulin and the indwelling butterfly needle that resulted in lipohypertrophy. We are at present investigating the sensitivity to insulin of fat from various sites in this patient.

${ }^{1}$ Renold AE, Marble A, Fawcett DW. Action of insulin on deposition of glycogen and storage of fat in subcutaneous tissue. Endocrinology 1950; 46:55-61.

(Accepted 9 September 1982)

Metabolic Unit, Department of Chemical Pathology, Royal Free Hospital and School of Medicine, London NW3 1QG

A MIER, MB, MRCP, registrar

J WEERAKOON, SRN, charge nurse

P DANDONA, DPHIL, MRCP, director, metabolic unit, senior lecturer in chemical pathology

\section{Acute colitis due to Plesiomonas shigelloides}

The complexity of infective colitis is gradually becoming unravelled as more pathogens capable of causing colonic inflammation are identified. Salmonella, Campylobacter, Clostridium difficile, and, more recently, Aeromonas hydrophila ${ }^{1}$ have been recognised for their potential to cause colitis. There are still cases of self-limiting acute colitis with negative findings on bacteriology but which otherwise behave like infective colitis, ${ }^{2}$ clearly indicating the presence of other agents unrecognised for their ability to cause colitis. We report a case of acute colitis associated with Plesiomonas shigelloides, an agent long suspected to be a cause of acute diarrhoea in man.

\section{Case report}

In late September 1981, a 41-year-old Englishman developed acute diarrhoea and vomiting. The stools were of small volume, green and slimy, mixed with blood, and averaging 10 to 15 a day. He had colicky abdominal pains relieved by defecation. His wife also had loose motions at about the same time but her symptoms abated after two days. The man had not been abroad recently. He was a keen fisherman and was fishing downstream from a sewage farm two days before the onset of his illness. He had had no previous bowel problems.

After nine days of unremitting symptoms he was admitted to hospital. On examination the abdomen was generally tender but soft. Sigmoidoscopy revealed an inflamed oedematous rectal mucosa that bled easily. Rectal biopsy showed acute inflammatory infiltration in the lamina propria, somewhat patchy in character, and occasional crypt abscesses. There was hyperplasia of crypts but the architecture was not distorted. The features were consistent with infective colitis. Stool cultures grew $P$ shigelloides in almost pure culture, but no other recognised bowel pathogens could be identified and virological study gave negative results.

The patient's symptoms settled spontaneously four days after admission and he has remained well since. Mucosa appeared normal on repeat sigmoidoscopy two months later, but the rectal biopsy showed persisting patchy inflammation and crypt abscesses and a rather heavy infiltration of the lamina propria by mononuclear cells, mainly plasma cells. The overall appearance was of infective colitis. A barium enema performed at that time showed no abnormalities. Histological examination of a further rectal biopsy specimen in March 1982 showed no abnormality.

\section{Comment}

$P$ shigelloides has interested medical microbiologists since 1947, when it was discovered that the organism had an $\mathrm{O}$ antigen that was identical with that of phase 1 Shigella sonnei. ${ }^{3}$ Since then it has been implicated as an aetiological agent in sporadic cases and outbreaks of diarrhoea in various parts of the world. $P$ shigelloides was isolated in pure culture of stools of patients with diarrhoea but otherwise healthy. A recent ecological study from Tokyo revealed wide distribution with not infrequent isolations from dogs, cats, freshwater fish, river water, 